(2) Open Access Full Text Article

\title{
Preparing for the National Health Service: the importance of teamwork training in the United Kingdom medical school curriculum
}

This article was published in the following Dove Press journal:

Advances in Medical Education and Practice

\author{
Abhinaya Chandrashekar \\ Jenanan Mohan \\ Imperial College London Faculty of \\ Medicine, Imperial College London, \\ London, UK
}

\begin{abstract}
Doctors are required to work in teams every day at every stage in their careers. In the United Kingdom (UK), with a drive towards an integrated healthcare system, teamwork has become a major focus amongst healthcare professionals and their skill set must reflect this. For doctors, the art of teamwork needs to be developed from the early stages of training, in order to minimise fragmentation of care and its detrimental impact on patients. The World Health Organisation emphasises the importance of doctors adopting a multi-disciplinary team approach, yet amongst medical students, collaborative work is often disregarded. Fundamentally, the system that produces future doctors overlooks the importance of teamwork. Therefore, the undergraduate curriculum must be reshaped to embed teamwork within its principles. Future doctors will thus be equipped with lifelong abilities to collaborate closely amongst peers in order to deliver care holistically. Adapting medical school curricula across the UK will present inevitable challenges and these must be understood, in order to generate strategies that cultivate a culture of teamwork amongst the doctors of the future.
\end{abstract}

Keywords: teamwork, team-based learning, curriculum, undergraduate, medical education

\section{Introduction}

Teams form the foundation of any organisation. The concept of sharing ideas, distributing work and collaborating to solve a problem can be applied to any field of practice. In healthcare, teamwork is particularly vital in delivering continuity of care. In the UK, with a rising prevalence of patients with chronic conditions that need multiple dimensions of care, healthcare professionals (HCPs) must be able to work in teams across disciplines. ${ }^{1}$ One such chronic condition would be diabetes. Diabetes is not just managed by an endocrinologist but requires a vast multidisciplinary team (MDT), including nurses, dieticians, ophthalmologists, pharmacists, GPs and community workers, all from various backgrounds. Patients engage with each of these professionals with the hope that collectively, all aspects of their care are addressed. ${ }^{2}$ However, only by these professionals all collaborating and sharing information can this care be provided holistically, and better patient outcomes be achieved. ${ }^{3}$ Importantly, this is just one example amongst a multitude of chronic conditions that are best managed when teamwork is performed effectively.

With a drive towards a patient-centric National Health Service (NHS), there is a growing need to create teams that communicate well in order to provide both health and social care on all levels. On a national level, teams traverse organisations such
Correspondence: Abhinaya

Chandrashekar

Imperial College London Faculty of

Medicine, Imperial College London,

London SW7 2DD, UK

Email abhinaya.c.shekar@gmail.com 
as the Department of Health and NHS England, to ensure the regulation and implementation of health policies in the best interests of the public. ${ }^{4}$ The NHS Five Year Forward View aims to generate further collaboration between care providers, through creating models such as primary and acute care systems (PACs) and multispecialty community providers (MCPs) in order to reap the benefits of care integration. ${ }^{5}$ On an organisational level, a well-functioning MDT enables HCPs to seek informed opinions during decision-making, as well as spread responsibility and pressure in challenging medical scenarios. ${ }^{6,7}$ However, successful MDTs require successful team members, who have honed skills such as communication, negotiation and respect. ${ }^{8}$ These skills need to be nurtured from the foundations of practice.

Whilst medical school prepares future doctors for academic complexities and how to manage clinical scenarios alone, there is less focus on how to be a good team member. ${ }^{9}$ Banerjee et al found that despite high face validity, medical schools have traditionally not included the specific knowledge, skills and attitudes required to function within an MDT. ${ }^{9}$ Whilst students are taught how to communicate with patients, there is far less consideration on how to communicate with peers.

As fifth year medical students with a Bachelor's in Management, we have been taught invaluable teamwork skills. The concept of collaborating with peers in order to achieve success was embedded into the structure of the Management course and as a result, shed light on the significance of teamwork training during medical school.

Rather than waiting for these skills to develop in situ during clerkships, ${ }^{10}$ the undergraduate curriculum must incorporate teamwork and team-based learning (TBL) to a greater degree, in order to equip future doctors with the skills required to work within an MDT in the NHS.

\section{What does teamwork mean?}

Teamwork is defined as "the combined action of a group, especially when effective and efficient." ${ }^{11}$ It involves the blending of different working cultures which is achieved through the sharing of skills and knowledge. ${ }^{12}$ Within healthcare, teamwork is multi-disciplinary; the appropriate utilisation of knowledge, skills and best practice protocol traverses service provider boundaries such as healthcare, social care and the private sector. ${ }^{13}$ This takes place at both a national and local level within the NHS. Almost 20 years ago, the then Secretary of State for Health called for cooperation and partnership at all levels of the NHS to ensure seamless patient-centred care takes place. ${ }^{14}$

The Royal College of Physicians outlines three key abilities team members must have when partaking in a group activity: technical competence, conceptual skills and interpersonal skills. ${ }^{3}$ Technical competence relates to the recognition of each team member's knowledge and strengths. Conceptual skills allow team members to plan, analyse and ultimately make decisions together. Finally, interpersonal skills involve an awareness of the needs of fellow colleagues and the ability to manage one's own emotions and those of other team members. ${ }^{3}$ This incorporates principles such as responsibility, communication, autonomy, trust and respect. ${ }^{12}$ In order to reap future benefits in patient care, these must be continually cultivated. ${ }^{3}$

\section{Benefits}

\section{Benefits to patients}

The benefits of teamwork to the quality of patient care has been well-documented. ${ }^{6,15,16}$ Sood identified 3 determinants of quality: patient safety, patient experience and clinical effectiveness, all of which can be positively impacted by successful teamwork. ${ }^{17}$

Patient safety can be defined as the "prevention of errors and adverse effects to patients". ${ }^{15}$ These errors often occur as a result of the miscommunication of information between staff and management of patients by different HCPs. ${ }^{18}$ The unfamiliarity of HCPs in a care setting is inevitable given the shift nature of work and the variable availability of staff. However, in order to minimise the effect of everchanging staff, teamwork skills should be a focal point of improvement. Effective teamwork can improve fluidity of information between HCPs, with staff knowing who from and when to seek help or relay information. ${ }^{19}$ As a result, continuity of care improves, reducing medical errors such as inconsistent treatment plans that result from poor communication. ${ }^{19}$ These benefits are reinforced by a multi-centre evaluation which identified a positive correlation between effective team behaviour, reduced clinical errors and improved staff attitudes. ${ }^{20}$

Patient experience encompasses aspects such as performance, reliability and serviceability, which are all greatly benefited by successful staff teamwork. ${ }^{17}$ The performance and reliability of a service can be evaluated by its punctuality and diagnostic accuracy. ${ }^{17}$ The NHS Cancer Plan demonstrated that effective MDT functioning can reduce the wait time from the first appointment to the start of 
treatment for lung cancer patients from 28 to 8 days. ${ }^{16}$ Furthermore, patients were found to report positive perceptions of prompt access to care, due to teamwork facilitating better care coordination between HCPs in hospitals, clinics and social care settings. ${ }^{16}$ Diagnostic accuracy can serve to reduce the emotional distress that patients experience of being transferred between incorrect departments. Effective communication can aid diagnostic accuracy by ensuring the right information is conveyed to the right individuals, thus reducing the number of unnecessary investigations. ${ }^{21}$ Serviceability pertains to speed, courtesy and competence. ${ }^{17}$ The courtesy and competence of HCPs is individually subjective and thus cannot always be impacted by teamwork. However, the speed of a service can be benefited in that the workload is dramatically reduced per individual and there is more confidence in decision making. ${ }^{19}$

Clinical effectiveness is defined as the "application of the best knowledge in order to achieve optimal patient outcomes". ${ }^{22}$ This includes generating the correct diagnosis, forming a subsequent patient-centric care pathway and improving patient mortality. ${ }^{23}$

Teamwork has been associated with sustained decreases in pre-operative delays, clinical decision time and adverse clinical events. This has led to improvements in patient outcomes, particularly patient mortality and morbidity. ${ }^{23}$ As a result of these benefits, American resuscitation guidelines now recommend teamwork education to be included in advanced life support courses, with teamwork deemed to be the single most important factor in minimising adverse events in patient transportation. ${ }^{24}$

\section{Benefits to the NHS}

On a national level, Sood states that in order to achieve integrated care and meet the needs of the defined population, collaboration between providers is essential. ${ }^{25}$ The benefits of such collaboration nationally can be categorised into efficient and effective practice.

Within the NHS, efficiency grossly describes reducing costs and increasing productivity. ${ }^{26}$ The Five Year Forward View reports on how integrated care can contribute to extensive healthcare cost savings and better allocation of healthcare resources. For example, in Greenwich, the local authority saved approximately $£ 1$ million by coordinating GP and social care services, which consequently reduced the number of people moving permanently into nursing care homes. ${ }^{27}$ This was achieved through clear communication to understand the needs and constraints of the local population and accordingly tailor care.

By transforming the term "productivity" into the concept of "reducing unwarranted variation of care", the significance of teamwork can be better perceived. ${ }^{26}$

The NHS' 10 Point Efficiency Plan outlines areas in which efficiency can or has been improved with the assistance of teamwork. For example, dealing with "minor" cases that present to the A\&E department was considered an area of high unproductivity. ${ }^{26}$ In a financially constrained healthcare system, the provision of inessential care to one, results in essential care being denied to another. By increasing collaboration between GPs and A\&E services, the NHS plans to redirect these cases to more out-of-hours GPs and urgent treatment centres, freeing up resources for more urgent cases. ${ }^{26}$ The possibility of such plans would not exist without inter-professional teamwork skills to coordinate these services.

Effective practice, amongst many factors, involves the reduction of medical errors and adverse events. ${ }^{28}$ These errors include medication errors, utilising inaccurate patient data and failure to diagnose or treat, which can have dramatic impacts on patient outcome. ${ }^{29}$ Almost half of such errors are preventable, with evidence to suggest most originate from human factors and teamwork. ${ }^{28}$

A particularly notable and poignant case that reached the public eye was the death of 8-year-old Victoria Climbié in 2002. Multiple communication and teamwork failures between 10 social care teams and services as well as 2 hospitals where she was admitted due to suspected deliberate harm, resulted in Victoria's preventable death from child abuse. ${ }^{30}$ The Laming Inquiry into this case outlined that there were at least 12 opportunities where individuals could have communicated across disciplines in order to successfully intervene in the life of Victoria. ${ }^{30}$

The impact of individual teamwork skills on patient management is thus profound and must not be underestimated. Empirical evidence suggests that improving these skills may be key to reducing medical errors, with particular focus on increasing teamwork assessment tools within the undergraduate medical curriculum. ${ }^{28}$ Only by preparing future doctors for scenarios where interdisciplinary communication can save lives, can advances be made towards achieving effective practice.

\section{Benefits to HCPs}

Studies have reinforced the notion that members of the MDT also gain positive benefits when an ethos of working 
together is instilled. ${ }^{6,31,32}$ Teamwork has been shown to enhance both the physical and mental wellbeing of team members, ${ }^{6}$ crucial for HCPs in the NHS working under intense pressure. A study conducted amongst 50,000 primary care workers showed that teamwork increased health and safety in the workplace, with fewer staff suffering injuries at work. ${ }^{31}$ Poignantly, staff who worked together as a team were less likely to experience bullying, harassment, abuse or physical violence. ${ }^{31}$ These effects serve to indicate the impact of functioning teams on lowering levels of stress amongst staff members. ${ }^{32}$

Another positive aspect of teamwork is that greater clarity is given about the job roles of each member. ${ }^{6}$ In a team, members all prescribe to common goals. Each team member has clear expectations on what they need to accomplish. ${ }^{15}$ This clarity means that the team as a whole can take advantage of the division of labour, achieving more than the sum of its part and avoiding role overlap. ${ }^{33}$ With role conflict being a contributing factor to a lower job satisfaction amongst staff, a team approach in healthcare is essential. ${ }^{32}$

\section{Benefits to students}

The effect of group work on students has been reviewed extensively, with favourable scientific support. ${ }^{34,35}$ TBL was found to promote individual and group accountability as well as collaborative skills. Students were found to learn to "inquire, share ideas, problem solve and construct new understandings", and were more motivated in group settings compared to working alone. ${ }^{34}$ Furthermore, over time, individuals were found to recognise the strengths of others and generate internal personal changes in order to maximise positive results in decision-making. ${ }^{35}$ The early development of these skills can serve to generate better interpersonal relationships in future clinical practice.

Academically, despite fears of being burdened by less able peers, it is evidenced to suggest that a TBL approach can vastly improve students' knowledge in comparison to traditional methods. ${ }^{35-37}$ In 2017, Doshi identified that medical students demonstrated increased satisfaction and scored $15.6 \%$ higher on average on topics assessed via TBL compared to other methods. ${ }^{38}$ Furthermore, it was found that when working on complex case studies, a greater depth of understanding was achieved with TBL compared to working independently. ${ }^{35}$ This could perhaps be due to TBL focusing on learning from peers and discussing concepts, rather than individually recalling facts.
The latter is often the result of the independent learning or traditional lecture style format. ${ }^{37}$

One can draw parallels between these complex cases and those of patients with significant comorbidities, in that a team-based approach is vital in reaching an optimal outcome. The fact that teamwork is regarded as paramount amongst practising physicians yet is somewhat disregarded amongst students suggests the need for its early integration within training. ${ }^{39}$ Teamwork skills must be developed at medical school, where a safe environment can be provided, and student actions would not compromise patient safety. ${ }^{40}$

\section{Challenges}

Despite evidenced benefits, there is still little teamwork focus in medical schools in the UK. ${ }^{41}$ The General Medical Council (GMC) thoroughly stipulates a list of outcomes that all undergraduate medical students are required to meet by the end of university, and state that medical schools are responsible for facilitating these outcomes. Within interprofessional education and team learning, the following guidance is provided (Figure 1). ${ }^{42}$

However, not enough emphasis is placed on teaching, assessing and ensuring all of these outcomes are achieved.

Firstly, some of these outcomes need to be taught during medical school training. For example, working effectively as a team by " referring to colleagues for investigations and advice" and "safely passing on information using clear and appropriate communication" are two outcomes deemed vital by the GMC, ${ }^{42}$ yet not all medical students are taught how to write referral letters. The clarity of referral letters are of paramount significance when asking colleagues for advice and further investigations for a patient, and overall, in ensuring effective communication between care providers in the NHS. Without the necessary information, patient care can be disjointed and inadequate. ${ }^{61}$ Neither the structure nor the basic content required is taught at all medical schools, and without proper teaching, future teamwork can be impacted, as students may not know how to communicate with other HCPs when requiring further opinions on patient care.

Secondly, assessment of these teamwork requirements through standardised assessments of across medical schools is limited. Currently, the only formal standardised exam sat by all UK medical students that assesses teamwork is the Situational Judgement Test. ${ }^{41}$ However, even within that exam, answers are based upon "how a student reports they would act rather than from a direct assessment of their behaviour." ${ }^{41}$ Without a direct assessment of 
9 Newly qualified doctors must learn and work effectively within a multi-professional and multi-disciplinary team and across multiple care settings. This includes working face to face and through written and electronic means, and in a range of settings where patients receive care, including community, primary, secondary, mental health, specialist tertiary and social care settings and in patients' homes.

They myst be able to:

a Demonstrate their contribution to effective interdisciplinary team working with doctors from all care settings and specialties, and with other health and social care professionals for the provision of safe and high-quality care

b Work effectively with colleagues in ways that best serve the interests of patients. This includes:

Safely passing on information using clear and appropriate spoken, written and electronic communication:

At handover in a hospital setting and when handing over and maintaining continuity of care in primary, community and social care settings

When referring to colleagues for investigations or advice

When things go wrong, for example when errors happen

- Questioning colleagues during handover where appropriate

Working collaboratively and supportively with colleagues to share experiences and challenges that encourage learning

Responding appropriately to requests from colleagues to attend patients

Applying flexibility, adaptability and a problem-solving approach to shared decision making with colleagues.

C Recognise and show respect for the roles and expertise of other health and social care professionals and doctors from all specialties and care settings in the context of working and learning as a multiprofessional team.

Figure I List of General Medical Council outcomes pertaining to teamwork among medical graduates.

Note: Reproduced with permission from General Medical Council (GMC). Outcomes for graduates 2018. 695 Available from: https://www.gmc-uk.org/-/media/documents/ dcI I326-outcomes-for-graduates-2018_pdf-75040796.pdf. Accessed January 12, 2019. @ 2018 General Medical Council. ${ }^{42}$

behaviour, these teamwork qualities are not formally tested, and thus how can it be ensured that they exist amongst future doctors? More importantly, as a consequence of not certifying a baseline level of teamwork skills, the NHS cannot then expect to have future employees who are all effective team workers.

The third challenge impacting future teamwork is the mentality of medical students. The GMC guidelines stipulate the importance of "working collaboratively and supportively with colleagues to share experiences and challenges that encourage learning". ${ }^{42}$ In order to achieve this outcome, a collaborative environment within medical school must be created where ideas are shared and discussed. However, it can be argued that instead, medical school fosters a paradoxical environment, instilling a competitive attitude right from the beginning. The process of applying for admission is incredibly competitive; for example, the ratio of applicants versus admissions is 9:1 for Imperial College School of Medicine. ${ }^{43}$ Right from the time of application, a "lone physician" mindset is created, as students strive to outcompete peers. ${ }^{44}$ Throughout training, this mentality is further reinforced as students are consistently ranked against fellow medical students each year and assessed in both academic and practical examinations situations alone with full responsibility for the consequences. This individualistic and competitive environment somewhat contradicts the collaborative environment described by the GMC, and inevitably results in few tasks delegated to others. This perpetuates till the end of medical school, as individual examination rankings against peers are what decide where medical students are ultimately placed for their foundation year jobs. ${ }^{44,45}$ With little benefit in collaborating with others at medical school, students can develop a thought process in which help is not sought, ideas are not questioned and decisions are made alone. In the future, this mentality can echo within the workplace, with doctors not seeking advice nor sharing experiences with colleagues, detrimentally impacting both multidisciplinary teamwork and patient care.

Reinforcing this, when analysing what competencies were most important, there was a striking difference between where 
physicians and medical students placed teamwork. Physicians ranked teamwork as the second most important competency after responsibility, whereas students only placed it fifth. ${ }^{33}$ With the competitive and individualistic nature of medical school, it can be understood as to why teamwork is considered to be of less value despite its benefits.

The NHS wanting team workers yet medical schools honing competitive students that are not fully taught and assessed on teamwork capabilities is paradoxical. It is evident that the system must change in order to create future doctors who are able to work together effectively. There is growing evidence that interprofessional education should be provided for all health and social care students during their pre-clinical years. ${ }^{46}$ Students who have engaged in this often realise the importance and relevance of being able to working effectively with colleagues from other professions. ${ }^{46}$ If the NHS is to push towards a successful integrated healthcare system, interprofessional education must become a fundamental aspect of the medical curriculum in order to develop the attitudes, knowledge and skills required. ${ }^{47}$

\section{Integrating teamwork into the medical school curriculum}

Nationally, there have been efforts to improve teamwork amongst medical school graduates. The Centre for the Advancement of Interprofessional Education (CAIPE), who define the concept of interprofessional collaborative practice as "working in a team in a healthcare context", produced guidelines on how to introduce interprofessional education (IPE) at medical schools across the UK. ${ }^{62}$ An example of effective IPE that CAIPE highlighted was at the University of Teesside, which had included IPE education in all health and social care pre-registration curricula. Students interacting and collaborating with other HCPs from their 1st year of university allowed them to develop better communication skills and focus on skills to improve patient safety. ${ }^{62}$

Internationally, there is further literature exploring IPE and its effectiveness. Guraya and Barr conducted a systematic review on 12 pre-post original studies from the USA, Canada and UK, analysing the effectiveness of IPE using quantitative analysis. ${ }^{63}$ From this, they found that IPE had a positive effect on teamwork in 11 out of the 12 studies reviewed. One study included in the review analysed a PBL module involving students from medicine, nursing, pharmacy and occupational therapy. This study conducted by Eccott el al. discovered that after this learning module, the students had "increased knowledge about roles and perspectives, greater confidence to collaborate, and more motivation to engage in intra-curricular IPE". 63

In the USA, a different study focused on the usefulness of a simulation-based curriculum to introduce key teamwork principles to entering medical students at Vanderbilt University School of Medicine. ${ }^{64}$ In each group, each student was given different briefs and information about a particular scenario. The students would then have to work together to complete each task given to them. The study found that the simulation was useful for the medical students and they were able to develop better decision-making skills, as well as understand the importance of teamwork. ${ }^{64}$

These international studies serve as examples of successful interprofessional education efforts, and importantly, demonstrate ideas by which to improve teamwork training which can be integrated into medical schools in the UK.

Looking at the existing medical curricula in the UK, there have also been endeavours to introduce some aspects of teamwork, such as TBL quizzes, group enquiry based learning and clinical simulations. ${ }^{43,48,49}$ However, these are integrated to significantly varying degrees across UK universities. The University of Manchester Medical School predominantly adopts a problem-based learning (PBL) approach to medical education with a high teamwork focus, compared to the University of Oxford which offers a traditional approach with a stronger emphasis on independent learning. ${ }^{50,51}$ This poses an issue when standardising teamwork skills amongst all future doctors.

The solution must address the creation of teamwork skills within every medical student in every year. Therefore, we offer 3 suggestions by which one can integrate teamwork into the medical school curriculums and discuss the feasibility in doing so.

These are:

- Integration of "Management" focused intercalated degrees

- Stipulation of a set percentage of each academic year to be assessed via TBL methods

- Introduction of MDT case scenarios for medical students in clinical years

\section{Integration of "management" focused intercalated degrees}

Amongst intercalated degrees, Management or Healthcare Management is only offered within five medical schools, 
representing $15 \%$ of bodies awarding medical degrees in the UK. ${ }^{52,53}$ As a course, management incorporates significant elements of teamwork as modules focus around understanding the strengths and weaknesses of others in order to allocate leadership roles and optimise outcomes. These are key qualities medical students should have before working within a future clinical MDT. ${ }^{15}$ At Imperial College Business School, $49 \%$ of the Management degree consists of collaborative work, illustrating the extent of emphasis placed on teamwork in order to achieve a successful result. ${ }^{54}$ In clinical practice, a successful result translates as an optimal patient outcome.

However, the solution is not necessarily to generate a new teamwork-focused intercalated degree at every medical school. There are inevitable cost implications with setting up a new course. Additionally, it is not compulsory to neither intercalate at every medical school, nor intercalate specifically within a degree focused on group work. ${ }^{52,55}$ Thus, demand relies on students actively choosing these courses. If there is insufficient interest, the course may not be feasible to run every year. Therefore, by implementing a new degree, there is no guarantee that all medical students cover teamwork skills within their undergraduate course, which is our primary focus.

\section{A set percentage of each academic year assessed via TBL methods}

The GMC stipulates that leadership and teamwork must be part of the standards and outcomes of medical school training for all students. ${ }^{56}$ Additionally, in guidelines for provisionally registered doctors, it is a mandatory requirement that clinicians are able to work effectively within a team. ${ }^{53}$ However, there is little guidance on how exactly to ensure that this takes place, and thus there is inevitable variability in how much group work is incorporated across medical schools. Therefore, to even out discrepancies, we propose that a percentage (eg $20 \%$ ) of each overall year must be assessed via TBL methods across all schools. This should be emphasised by the GMC to be a national minimum requirement, such that regardless of the course structure, teamwork skills are assessed. This could be in the form of an entire exam conducted in a group, or via a portion of each end-of-year assessment involving team-based activity.

This integration of TBL would introduce teamwork with groups of varying sizes into the medical curriculum. Reflecting on personal experience, group work ranging from case studies in pairs to large group presentations all taught different skills. In pairs, one learns how to debate and make decisions without a "majority vote", whereas in bigger groups, one learns how to distribute a large workload evenly whilst maximising the strengths of individuals present. Therefore, medical schools should continue to encourage individuals to perform tasks such as case presentations and mock examinations in pairs and larger groups, such that students can learn collaboration skills as well as rely on each other for ideas and support. ${ }^{57}$ These may either form part of an assessment or may be sporadic learning opportunities.

\section{Introduction of MDT case scenarios for medical students in clinical years}

Within an MDT, it is also vital to know the roles of each member. With an immense workload and time constraints, knowing which MDT members to rely upon and how to share jobs is crucial for both the doctor's mental health and the patient's wellbeing. ${ }^{6}$ Therefore, we propose the introduction of MDT case scenarios for medical students in clinical years. Case scenarios have been described to be beneficial in reinforcing knowledge as well as developing clinical reasoning, by simulating a real-life scenario without consequences. ${ }^{58,59}$ Simulations are recommended for all doctors to experience by the NHS Foundation Programme, teaching teamwork skills and patient management protocols. ${ }^{60}$ Active immersion by playing the role of each individual involved in a typical scenario such as a cardiac arrest, would allow students to manage complex cases by utilising the capabilities of all HCPs present. ${ }^{46}$ As simulations are difficult to academically assess, these should be integrated as enjoyable learning opportunities for students.

With a growing focus on integrated care in the NHS, an ethos of teamwork needs to be embedded into every aspect of healthcare. The benefits of collaboration are evident through the success of MDTs in providing seamless patient-centric care, yet the focus must be on the individual skills that form the foundation of these teams. It is important to emphasise that though this review focuses on medical students, these skills are important amongst all professionals involved in patient care, and the analysis of how to improve teamwork amongst other HCPs poses as future scope for this paper. Overall, teamwork qualities are those that must be continually honed and practiced. For future doctors, being equipped with these teamwork skills during medical school will prepare them for the complex networks and partnerships created in an ever-changing NHS.

\section{Acknowledgments}

We would like to thank Dr Edgar Meyer and Dr Harpreet Sood for their continual guidance and support in this work. 


\section{Disclosure}

There are no financial or other conflicts of interest in the making of this article.

\section{References}

1. The King's Fund. Long-term conditions and multi-morbidity. Available from: https://www.kingsfund.org.uk/projects/time-think-dif ferently/trends-disease-and-disability-long-term-conditions-multimorbidity. Accessed January 25, 2019.

2. Centers for Disease Control and Prevention (CDC). Team care approach for diabetes management Available from: https://www. cdc.gov/diabetes/ndep/pdfs/ppod-guide-team-care-approach.pdf. Accessed January 20, 2019.

3. The Royal College of Physicians. Improving teams in healthcare: resource 1- building effective teams. Available from: https://www. rcplondon.ac.uk/projects/outputs/improving-teams-healthcareresource-1-building-effective-teams. Accessed November 12, 2018.

4. Care Quality Commission (CQC). Joint framework: commissioning and regulating together: a practical guide for staff; 2018. Available from: https://www.cqc.org.uk/sites/default/files/20180108_CQC_ NHSE_NHSCC_joint_working_framework.pdf. Accessed November 4, 2018 .

5. The King's Fund. Primary and acute care systems. Available from: https://www.kingsfund.org.uk/projects/nhs-five-year-forward-view/ primary-acute-care-systems. Accessed November 9, 2018.

6. Babiker A, El Husseini ME, Al Nemri A, et al. Health care professional development: working as a team to improve patient care. Sudan $J$ Paediatrics. 2014;14(2):9-16. Available from: https://www.ncbi.nlm. nih.gov/pmc/articles/PMC4949805/ Accessed November 4, 2018.

7. Firth-Cozens J. Multidisciplinary teamwork: the good, bad, and everything in between. BMJ Qual Saf. 2001;10(2):65-66. doi:10.1136/ qhc. 10.2 .65

8. Bridges D, Davidson R, Soule Odegard P, Maki I, Tomkowiak J. Interprofessional collaboration: three best practice models of interprofessional education. Med Educ Online. 2011:16(1):6035. doi:10.3402/meo.v16i0.6035

9. Banerjee A, Slagle JM, Mercaldo ND, et al. A simulation-based curriculum to introduce key teamwork principles to entering medical students. Available from:. BMC Med Educ. 2016;16(1):295. doi:10.1186/s12909-016-0808-9

10. Ofstad W, Brunner L. Team-based learning in pharmacy education. Am J Pharm Educ. 2013;77(4):70. doi:10.5688/ajpe77470

11. Oxford Dictionaries. Definition of teamwork in English. Available from: https://en.oxforddictionaries.com/definition/team work. Accessed November 3, 2018.

12. Bridges D, Davidson R, Soule Odegard P, Maki I, Tomkowiak J. Interprofessional collaboration: three best practice models of interprofessional education. Med Educ Online. 2011;16(1):6035. doi:10.3402/meo.v16i0.6035

13. National Health Service (NHS). MDT development - working toward an effective multidisciplinary/multiagency team; 2015. Available from: https://www.england.nhs.uk/wp-content/uploads/2015/01/mdtdev-guid-flat-fin.pdf. Accessed January 17, 2019.

14. Barr H. Interprofessional education- today, yesterday and tomorrow. higher education academy, learning \& teaching support network for health sciences \& practice, occasional paper 1;2002. Available from: https:/www.caipe.org/resources/publications/caipe-publications/ caipe-2002-interprofessional-education-today-yesterday-tomorrowbarr-h. Accessed January 23, 2019.

15. World Health Organisation (WHO). Topic 4: being an effective team player. WHO Patient Safety Curriculum Guide Med Schools. 2009. Available from: http://www.who.int/patientsafety/activities/technical/ who_ps_curriculum.pdf. Accessed November 12, 2018.
16. National Health Service (NHS). The NHS cancer plan; 2000. Available from: https://www.thh.nhs.uk/documents/_Departments/ Cancer/NHSCancerPlan.pdf. Accessed 28 November 2018.

17. Sood H. Quality and Quality Improvement. [lecture] Managing Challenges in Healthcare Organisations. London: Imperial College London. February 4, 2018.

18. Petersen LA, Brennan TA, O’Neil AC, Cook EF, Lee TH. Does housestaff discontinuity of care increase the risk for preventable adverse events? Ann Intern Med. 1994;121(11):866-872. Accessed November 14, 2018. doi:10.7326/0003-4819-121-11-199412010-00008

19. Vermeir P, Vandijck D, Degroote S, et al. Communication in healthcare: a narrative review of the literature and practical recommendations. Int $J$ Clin Pract. 2015;69(11):1257-1267. doi:10.1111/ijcp.12686

20. Morey JC, Simon R, Jay GD, et al. Error reduction and performance improvement in the emergency department through formal teamwork training: evaluation results of the medteams project. Health Serv Res. 2002;37(6):1553-1581. doi:10.1111/1475-6773.01104

21. Tremblay D, Roberge D, Touati N, Maunsell E, Berbiche D. Effects of interdisciplinary teamwork on patient-reported experience of cancer care. BMC Health Serv Res. 2017;17(1):218. doi:10.1186/s12913017-2166-7

22. Southport and Ormskirk Hospital NHS Trust. Clinical effectiveness strategy (Clinical audit/research) 2013-2015. Available from: https:// www.southportandormskirk.nhs.uk/pdfs/Clinical-EffectivenessStrategy.pdf. Accessed November 30, 2018.

23. Weaver SJ, Dy SM, Rosen MA. Team-training in healthcare: a narrative synthesis of the literature. BMJ Qual Saf. 2014;23(5):359372. doi:10.1136/bmjqs-2013-001848

24. Mileder LP, Schmölzer GM. The impact of teamwork training on clinical practice and patient health. Am J Med. 2014;127(8):29. doi:10.1016/j.amjmed.2014.02.041

25. Sood H. Integrated Care. [lecture] Managing Challenges in Healthcare Organisations. London: Imperial College London; January 21, 2018.

26. National Health Service (NHS). Funding and Efficiency. Available from: https://www.england.nhs.uk/five-year-forward-view/next-stepson-the-nhs-five-year-forward-view/funding-and-efficiency/. Accessed January 22, 2019

27. National Health Service (NHS). Five year forward view. Available from: https://www.england.nhs.uk/wp-content/uploads/2014/10/5yfvweb.pdf. Accessed November 22, 2018.

28. Freytag J, Stroben F, Hautz WE, et al. Improving patient safety through better teamwork: how effective are different methods of simulation debriefing? Protocol for a pragmatic, prospective and randomised study. BMJ Open. 2017;7:e15977. doi:10.1136/bmjopen-2017-015977

29. National Health Service (NHS). One in 20 hospital deaths preventable. Available from: https://www.nhs.uk/news/medical-practice/onein-20-hospital-deaths-preventable/. Accessed January 22, 2019.

30. Laming WH The Victoria Climbié inquiry. Available from: https:// assets.publishing.service.gov.uk/government/uploads/system/ uploads/attachment_data/file/273183/5730.pdf. Accessed January $18,2019$.

31. West M Developing cultures of high quality care. [Presentation] The King's Fund. March 7, 2013. Available from: https://www.kingsfund. org.uk/sites/default/files/michael-west-developing-cultures-\%20highquality-care-kingsfund-feb13.pdf. Accessed November 28, 2018.

32. Jaruseviciene L, Liseckiene I, Valius L, Kontrimiene A, Jarusevicius G, Lapão LV. Teamwork in primary care: perspectives of general practitioners and community nurses in Lithuania. BMC Fam Pract. 2013;14:118. doi:10.1186/1471-2296-14-118

33. Kroposki M, Murdaugh C, Tavakoli A, Parsons M. Role clarity, organizational commitment, and job satisfaction during hospital reengineering. Nursingconnections. 1999;12(1):27-34. Available from: https://www. ncbi.nlm.nih.gov/pubmed/10401399 Accessed 28 November 2018. 
34. Hammar Chiriac E. Group work as an incentive for learning - students' experiences of group work. Front Psychol. 2014;5. doi:10.3389/ fpsyg.2014.00558

35. Michaelsen L, Sweet M, The essential elements of team-based learning. New Directions Teach Learn. 2008;2008(116):7-27. Available from: https://onlinelibrary.wiley.com/doi/abs/10.1002/t1.330 Accessed 12 November 2018. doi:10.1002/tl.v2008:116

36. Lein D, Lowman J, Eidson C, Yuen H. Evaluation of team-based learning in a doctor of physical therapy curriculum in the United States. $J$ Educ Eval Health Prof. 2017;14:3. doi:10.3352/jeehp.2017.14.3

37. Bleske B, Remington T, Wells T, Klein K, Tingen J, Dorsch M. A randomized crossover comparison between team-based learning and lecture format on long-term learning outcomes. Pharmacy. 2018;6 (3):81. doi:10.3390/pharmacy6030081

38. Doshi N. Effectiveness of team-based learning methodology in teaching transfusion medicine to medical undergraduates in third semester: a comparative study. Asian J Transfus Sci. 2017;11(2):87. doi:10.4103/ajts.AJTS_123_16

39. Ennen CS, Satin AJ. Training and assessment in obstetrics: the role of simulation. Best Pract Res Clin Obstetrics Gynaecol. 2010:24 (6):747-758. Available from. doi:10.1016/j.bpobgyn.2010.03.003

40. Fürstenberg S, Harendza S. Differences between medical student and faculty perceptions of the competencies needed for the first year of residency. BMC Med Educ. 2017;17(1). doi:10.1186/s12909-017-1036-7

41. Parker R, Hodierne L, Anderson E, Davies R, Elloy M. Academic ability and teamworking in medical students. Clin Teach. 2018;15:15. doi:10.1111/tct.12800

42. General Medical Council (GMC). Outcomes for graduates 2018. Available from: https://www.gmc-uk.org/-/media/documents/ dc11326-outcomes-for-graduates-2018_pdf-75040796.pdf. Accessed January 12, 2019.

43. Imperial College London. MBBS/BSc medicine. Available from: https://www.imperial.ac.uk/study/ug/courses/school-of-medicine/med icine/. Accessed January 17, 2019.

44. Saba GW, Villela TJ, Chen E, Hammer H, Bodenheimer T. The myth of the lone physician: toward a collaborative alternative. Ann Fam Med. 2012;10(2):169-173. doi:10.1370/afm.1353

45. Medical Schools Council. Foundation Programme. Available from: https://www.medschools.ac.uk/studying-medicine/after-medicalschool/foundation-programme. Accessed November 12, 2018.

46. Barr H, Gray R, Helme $M$, et al. Interprofessional education guidelines 2016. Available from: https://www.caipe.org/ resources/publications/caipe-publications/barr-h-gray-r-helme-mlow-h-reeves-s-2016-interprofessional-education-guidelines. Accessed January 23, 2019.

47. Barr H. Interprofessional education- the genesis of a global movement. Available from: https://www.caipe.org/resources/publications/ caipe-publications/caipe-2015-interprofessional-education-genesisglobal-movement-barr-h. Accessed January 23, 2019.

48. Khogali SE, Smithies AD, Gray A, Manca A, Lafferty NT Team-based learning in a UK medical school: using mobile friendly technology to support the in-class individual readiness assurance test. Proceedings of the European Conference on e-Learning (ECEL), 2014; Copenhagen. Available from: https://www.researchgate.net/publication/266030802_ Team-Based_Learning_in_a_UK_Medical_School_Using_Mobile_ Friendly_Technology_to_Support_the_In-class_Individual_Readiness Assurance_Test Accessed 26th January 2019.
49. King's College London. Interprofessional Education (IPE). Available from: https://www.kcl.ac.uk/health/study/facilities/chantler/teaching/ ipe.aspx. Accessed January 26, 2019.

50. General Medical Council (GMC). Review of Manchester Medical School. Available from: https://www.gmc-uk.org/-/media/docu ments/Manchester_Medical_School_report_v6.0.pdf_55887809.pdf 60955384.pdf. Accessed January 12, 2019.

51. British Medical Association (BMA). Course and teaching types at medical school. Available from: https://www.bma.org.uk/advice/ career/studying-medicine/becoming-a-doctor/course-types. Accessed January 18, 2019.

52. Intercalate. Browse Courses. Available from: http://intercalate.hyms. ac.uk/courses.aspx .Accessed November 302018.

53. General Medical Council. Bodies awarding UK medical degrees. Available from: https://www.gmc-uk.org/education/how-we-qualityassure/medical-schools/bodies-awarding-uk-medical-degrees. Accessed 24 November 2018.

54. Imperial College Business School. Intercalated BSc 2017-18 Programme handbook. Available from: https://imperialbusiness. school/custom/uploads/2017/06/IBSc-Programme-Handbook-2017. 18.pdf. Accessed November 8, 2018.

55. Sonsale A, Bharamgoudar R. Equipping future doctors: incorporating management and leadership into medical curriculums in the United Kingdom. Perspect Med Educ. 2017;6(2):71-75. doi: $10.1007 / \mathrm{s} 40037-017-0327-3$

56. GMC outcomes for graduates. Available from: https://www.gmc-uk. org/-/media/documents/dc11326-outcomes-for-graduates-2018_pdf75040796.pdf. Accessed March 21, 2019.

57. Ofei-Dodoo S, Goeri K, Moser S. Exploring the impact of group size on medical students' perception of learning and professional development during clinical rotations. Kansas J Med. 2018;11(3):70-75. Available from: https://www.ncbi.nlm.nih.gov/pmc/articles/ PMC6122880/. Accessed 28 November 2018.

58. Abas T, Juma F. Benefits of simulation training in medical education. Adv Med Educ Pract. 2016;7:399-400. doi:10.2147/AMEP.S110386

59. Datta R, Upadhyay K, Jaideep C. Simulation and its role in medical education. Med J Armed Forces India. 2012;68(2):167-172. doi:10.1016/S0377-1237(12)60040-9

60. National Health Service (NHS). Foundation programme curriculum. Available from: http://www.foundationprogramme.nhs.uk/ sites/default/files/2018-07/Curriculum_0.pdf. Accessed January 22, 2019

61. The King's Fund. Referral management (Lessons for success). Available from: https:/www.kingsfund.org.uk/sites/default/files/ Referral-management-lessons-for-success-Candace-Imison-ChrisNaylor-Kings-Fund-August2010.pdf. Accessed May 30, 2019.

62. Barr H, Low H Introducing interprofessional education. Available from: https://www.caipe.org/resources/publications/caipe-publica tions/barr-h-low-h-2013-introducing-interprofessional-education13th-november-2016. Accessed May 23, 2019.

63. Guraya SY, Barr H. The effectiveness of interprofessional education in healthcare: a systematic review and meta-analysis. Kaohsiung J Med Sci. 2018;34(3):160-165. doi:10.1016/j.kjms. 2017.12.009

64. Banerjee A, Slagle JM, Mercaldo ND, et al. A simulation-based curriculum to introduce key teamwork principles to entering medical students. BMC Med Educ. 2016;16:295. doi:10.1186/s12909-0160808-9 


\section{Publish your work in this journal}

Advances in Medical Education and Practice is an international, peerreviewed, open access journal that aims to present and publish research on Medical Education covering medical, dental, nursing and allied health care professional education. The journal covers undergraduate education, postgraduate training and continuing medical education including emerging trends and innovative models linking education, research, and health care services. The manuscript management system is completely online and includes a very quick and fair peer-review system. Visit http://www.dovepress.com/testimonials.php to read real quotes from published authors.

Submit your manuscript here: http://www.dovepress.com/advances-in-medical-education-and-practice-journal 